\title{
An innovative method for anchoring glucose-sensing molecules on glassy micro-particles
}

\begin{abstract}
In this work a modified glassy micro-particles glucose sensor was developed applying a new and effective procedure to anchor glucose oxidase on glassy micro particles using urea medium. The resulting sensor, in the presence of glucose, generates a color development in 60 seconds working at room temperature. The intensity of the resulting color shows a straightforward relationship with the glucose concentration in the samples tested. The resulting biosensor was tested to demonstrate its sensibility on dissolutions of glucose and honey.
\end{abstract}

Volume 4 Issue 5 - 2020

\author{
Marianela Zoratti, María E Centurión, Marisa \\ A Frechero \\ Departamento de Química, Universidad Nacional del Sur, \\ Argentina
}

\begin{abstract}
Correspondence: Marisa A Frechero, Departamento de Química, INQUISUR (UNS-CONICET), Universidad Nacional del Sur, Av. Alem I253- Bahía Blanca, Buenos Aires, Argentina, Tel +542914595100,Email frechero@uns.edu.ar
\end{abstract}

Received: October 15,2020 | Published: October 26, 2020

\section{Introduction}

A device designed to sense a product of a biological interest is called biosensor. Commonly those devices could be used by specialists but, what is more important today is to design a sensor for no specialist operators. Those devices could also be used in an on-line automatized system. A transducer is needed for an automatized system application. This is a physical component that responds to a bio-product which is being sensing and is suitable to give an output signal that can be amplified, stored, or displayed. Those transducers can be grouped into four main classes: electrochemical, mass, optical and thermal. ${ }^{1}$ Among them, electrochemical detectors are very popular; however, they required a voltage to operate and, therefore, associated specific electrical instrumentation. Hence, if the molecule sensing is adequate to develop a color reaction, and then that color turns into a different color, a fundamental advantage emerges because, an electronic device is not needed and neither is a specialist technician. A biosensor is the fusion between a biological material and a physical transducer and its development is of interest in different fields of analytical chemistry. Much of the current biosensors are focused on the field of clinical diagnosis, environmental controls, pharmaceutical tests, for example. Among them, one of the greatest success has been the glucose biosensors. ${ }^{2,3}$ For enzymes and proteins is quite difficult to exchange electrons with electrode surfaces. This is because they usually have large and complex structures in which the redox centers are deeply immersed; then, the anchoring of proteins or enzymes on an electrode surface is a practical way to achieve an improved interfacial electron transfer. ${ }^{4}$ Nowadays, in order to develop miniaturized glucose oxidase (GOx) enzymatic biosensors for sensing glucose molecules (Glu) there is a need to use different green and affordable inorganic or organic substrates. ${ }^{5,6}$ The advantage of enzymatic methods is that they have high selectivity. While the GOx has high selectivity the disadvantage is its high cost when it is performed with the enzyme in solution. To overcome such inconvenience, it is necessary to re-use the enzyme. Several methods allow such strategy; for example, using a porous glass surface to immobilize the enzyme with a glutaraldehyde covalent bonding. Another method, is an ionic bonding on an ionic exchange resins, such as carboxymethyl, for chelate formation that allows the manufacture of micro-columns that can be coupled to a flow system reducing cost and avoiding a loss of selectivity and precision using immobilized enzymes on solid substrates. ${ }^{7}$
This requires a condensable precursor, often silanes of the type R'-Si(OR)3, with the functionality R' being stable under the conditions of the mesoporous silica synthesis. However, the typical silanes used as APTES or APTMEES (3-aminopropyltriethoxysilane or 3-aminopropyltris methoxy ethoxy ethoxy silane, respectively) are quite expensive and usually affects the pore lead to decreasing mesoscopic order. ${ }^{8} \mathrm{~W}$ Limbut et $\mathrm{al}^{9}$ proposed in 2004 a method to immobilize urease on three different support based on $\mathrm{SiO}_{2}$ for the determination of urea ${ }^{9}$ and they showed the high efficiency of such interaction. Therefore, in this work we propose an innovation in the traditional method ${ }^{10,11}$ for anchoring glucose-sensing molecules on an inorganic substrate using modified powdered silicate glasses as micrometric inner substrate, which is a very economical substrate and environmental friendly and anchoring a sensing molecules through an innovative and green chemical substance such is the urea. Followed by a procedure that allows to fix the GOx on the solid substrate sticking the sensing molecule without using expensive organic compounds through two coupled reactions: firstly, the glucose oxidase (GOD) catalyzes the oxidation of glucose to gluconic acid, generating $\mathrm{H}_{2} \mathrm{O}_{2}$ (Eq. 1); secondly, the hydrogen peroxide $\left(\mathrm{H}_{2} \mathrm{O}_{2}\right)$ is detected by a chromogenic oxygen acceptor: the phenol 4-aminophenazone (4-AF), in the presence of peroxidase (POD) (Eq. 2). Therefore, the intensity of the resulted color has a straightforward relationship with the glucose concentration in the sample carrying out a simple screening reaction to detect glucose concentrations greater than $0.4 \mathrm{~g} / \mathrm{L}$ through a color comparison.

$$
\begin{gathered}
\mathrm{C}_{6} \mathrm{H}_{12} \mathrm{O}_{6}+\mathrm{O}_{2}+\mathrm{H}_{2} \mathrm{O} \mathrm{GO} x \rightarrow \mathrm{C}_{6} \mathrm{H}_{12} \mathrm{O}_{7}+\mathrm{H}_{2} \mathrm{O}_{2} \\
2 \mathrm{H}_{2} \mathrm{O}_{2}+4-\mathrm{AF}+\mathrm{C}_{6} \mathrm{H}_{5} \mathrm{OH} \mathrm{POx} \rightarrow \mathrm{C}_{6} \mathrm{H}_{4} \mathrm{O}_{2}+4 \mathrm{H}_{2} \mathrm{O}
\end{gathered}
$$

In what follows, we show the procedure step by step and several test to evidence the success of each one. Then, the efficacy of the anchoring procedure to obtain a biosensor is tested, that can be used as a glucose detector in a honey aqueous dissolution.

\section{Experimental}

\section{Experimental techniques}

Solutions were prepared using tri-distilled water $(<1 \mu \mathrm{S})$. Urea (99,97\%, Mallinckrodt); glutaraldehyde solution grade II, $25 \%$ in $\mathrm{H}_{2} \mathrm{O}$ (Sigma-Aldrich); $0.1 \mathrm{M}$ phosphate buffer solution $\mathrm{pH} 6.8$; 
Enzymatic Glycemia (Wiener Lab): 25mM 4-aminophenazone dissolution in $0.92 \mathrm{M}$ Tris Buffer, $55 \mathrm{mM}$ phenol solution, glucose oxidase solution $(1000 \mathrm{U} / \mathrm{ml})$, peroxidase $(120 \mathrm{U} / \mathrm{ml})$ and $1 \mathrm{~g} / \mathrm{l}$ glucose solution. Nicolet Nexus FTIR instrument was used in order to obtain the FTIR absorption spectrum in the $2000 \mathrm{~cm}^{-1}-400 \mathrm{~cm}^{-1}$ range at room temperature. Samples were previously grounded to fine powders and dispersed thoroughly in $\mathrm{KBr}$. The amorphous character of the modified glass particles was tested by X-Ray Diffraction analysis (XRD) with a Bruker D8 Advance diffractometer in continuous scan mode $(\mathrm{Cu}$ anode, $45 \mathrm{kV}-30 \mathrm{~mA}$ at $298 \mathrm{~K}$ ) between $10^{\circ}<2 \theta<60^{\circ}$. Laser Scattering Particle Size and $\mathrm{Z}$ potential were done at room temperature in a Malvern Nano ZS90 equiment.

\section{Modified glassy micro-particles glucose sensor (MGGS)}

A commercial modified silicate glass with a high transition temperature $\left(\mathrm{T}_{\mathrm{g}}\right)$ was employed as inorganic solid substrates. An agate ball-milled was used to obtain micro-particles and the powder was sieved with a 150 mesh.

The small fraction of particles obtained after sieved with a 150 mesh was treated through the follow steps in order to generate the modified glassy micro-particles as a glucose sensor:

a) Glassy particles were stirred in a $\mathrm{NaOH} 1 \mathrm{M}$ dissolution for 24 hours at room temperature. Once such alkaline treatment was finished, the aqueous glass dispersion was filtered by gravity using Whatman $\mathrm{N}^{\circ} 3$ paper. The solid fraction retained on the paper was washed with tri-distilled water several times until obtaining a neutral flushing liquid. Therefore, the solid fraction was dried in an oven at $75^{\circ} \mathrm{C}$ for 3 hours in a regular atmosphere.

b) The resulting dried powder glass was then sonicated during 30 minutes $(160 \mathrm{~W})$ in a urea dissolution $(6.95 \mathrm{M})$. Therefore, the new dispersion system was again filtered, washed with distilled water and dried in an oven at $75^{\circ} \mathrm{C} 3$ hours in a regular atmosphere. This treatment allows to the glassy microparticles to have the adequate anchors on its surface.

c) The treated micro-particles glasses were stirred for 2 hours in a glutaraldehyde dissolution $(10 \% \mathrm{w} / \mathrm{w})$ at room temperature. Finally, this dispersion was filtered again through a Whatman $\mathrm{N}^{\circ} 3$ paper and washed several times with a phosphate buffer dissolution $\mathrm{pH} 6.8$ until the flushing liquid conserves the $\mathrm{pH}$. Finally, the decorated glassy particles were dry at $75^{\circ} \mathrm{C}$ for 3 hours in a regular atmosphere.

d) Finally, the anchor decorated glassy particles were mixed with the sensing molecules dissolution. At this time, we use Glycemia an enzymatic commercial product for glucose determination (Wiener lab) to guarantee the glucose molecules sensing response and to focus only on the evaluation of the new anchoring method efficiency. For this proposed, $1 \mathrm{~g}$ of anchor decorated glassy particles and $2 \mathrm{~mL}$ of the enzymatic commercial product were sonicated for $30 \mathrm{~min}$ in a cooling-gel bath to avoid heating to preserve the enzyme activity. In order, to finish the preparation process, the system was washed with phosphate buffer $\mathrm{pH} 6.8$ and carefully stored at $4^{\circ} \mathrm{C}$ in a closed jar to be effectively used during three months (Figure 1).

\section{Material characterization}

Figure 2 shows the FTIR spectrum of the silicate glass and the glassy micro-particles traded with urea dissolution after finished stage 2, i.e. glassy particles with where the N-H bonds are fixed on the glass surface and they are ready to receive the glutaraldehyde molecules. The bands observed are between $3500 \mathrm{~cm}^{-1}-3400 \mathrm{~cm}^{-1}$ and 1560 $\mathrm{cm}^{-1}-1640 \mathrm{~cm}^{-1}$ in the glassy micro-particles traded spectrum. Such response allows us to affirm that a successful anchor on the surface is reached. The set of bands between $800 \mathrm{~cm}^{-1}$ and $1080 \mathrm{~cm}^{-1}$ belongs to the vibration of silicon-oxygen bonds by flexion and stretching.

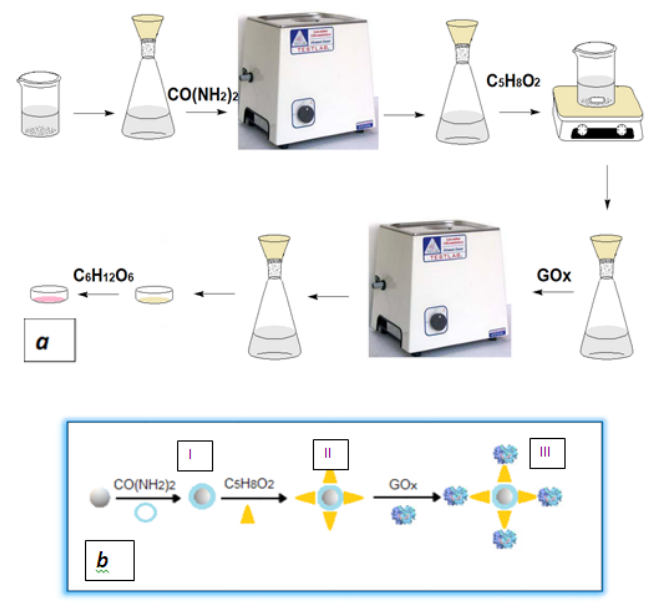

Figure I A) Scheme of preparation method; B) Method chemical reactions.
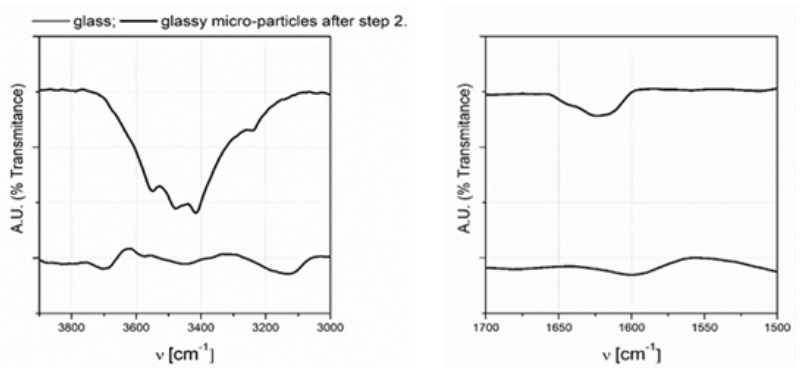

Figure 2 FTIR spectrum of the silicate glass and the glassy micro-particles traded with urea dissolution after finished stage 2 .

Figure 3 shows the glass particles X Ray diffraction patterns after the treatments 2 and 4 . From the results in this figure we confirm the absence of crystalline structures on the glass surface due to the fact that any peak appears.

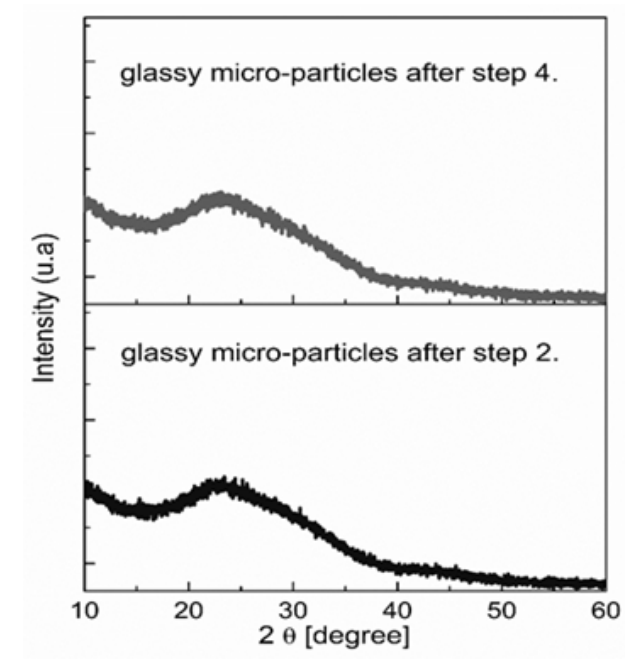

Figure $3 \times$ ray diffraction patterns of glassy particles after the treatments 2 and 4. 
Table I Charge density

\begin{tabular}{lll}
\hline Medium & ZP (mV) & $\begin{array}{l}\text { Zeta deviation } \\
(\mathbf{m V})\end{array}$ \\
\hline Water tri-distilled & -33.3 & 8.5 \\
0.IM Buffer phosphate $\mathrm{pH} \mathrm{7}$ & -16.9 & 0 \\
\hline
\end{tabular}

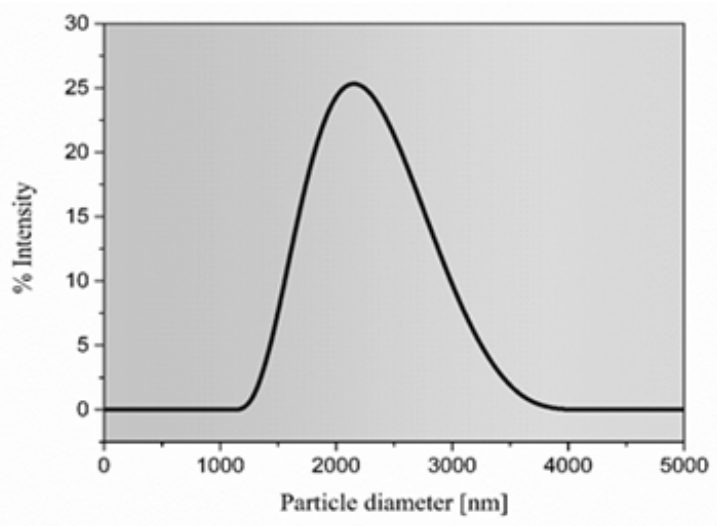

Figure $\mathbf{4}$ Laser scattering particle size distribution.

The adsorption phenomena on a surface is the ability of solid substrates to attract to some molecules from the medium in which it is in contact. Such phenomena can be physical or chemical, i.e. where the interactions are due to van der Waals forces or, in the second case, the interactions are electrostatic. Therefore, the surface fundamental property is its polarity. The property and the behavior of the substrate surface is strongly affected by the surface charge and, the Zeta potential $(\xi)$ is the indicator of surface charge and its measurement provides an understanding of its chemical behavior as a function of the medium, since the interaction with external ions depends both on the ions characteristics and on the surface characteristics. Considering the first step in our method we check the surface charge of the glass particles to ensure that optimum condition to allow the chemical reaction 3 and 4 that are explained in the next section. Table 1 shows the Zeta potential result where we confirm the optimal surface charge density ${ }^{12}$ and Figure 3 shows the size of glass particles distribution. Figure 4 shows the result obtained of the size particle distribution through the Laser Scattering Particle Size Analyzer and Table 1 of the charge density by the particles surface obtained by $\mathrm{Z}$ potential method.

\section{Application of the modified glassy micro- particles as a glucose sensor}

When a water film is on a glass surface two different chemical reactions are induced regulated by the $\mathrm{pH}$ of the solution. ${ }^{13,14}$ When the $\mathrm{pH}$ is lower than 9 a leaching process takes place and resulting ${ }^{15,16}$ in an ion-exchange where alkali ions from the glass are exchanged by $\mathrm{H}^{+}$or $\mathrm{H}_{3} \mathrm{O}^{+}$which is enhanced if the $\mathrm{pH}$ is higher than $9 .{ }^{17}$

$$
\begin{aligned}
& \sim \mathrm{Si}-\mathrm{ONa}+\mathrm{H}^{+} \leftrightarrows \mathrm{S} \mathrm{i}-\mathrm{OH}+\mathrm{Na}^{+} \\
& \sim \mathrm{Si}-\mathrm{ONa}+\mathrm{H}_{3} \mathrm{O}^{+} \leftrightarrows \mathrm{Si}-\mathrm{OH}+\mathrm{H}_{2} \mathrm{O}+\mathrm{Na}^{+}
\end{aligned}
$$

In a regulated $\mathrm{pH}$ condition those reactions are governed by the ion diffusion in the glass following the Fick's law until the steady state is reached and the thickness of the modified layer over the surface remains constant. Such hydrated silicate layer is itself a new amorphous phase as we evidence in Figure 3; that new phase allows the reaction with the urea given the covalent bond with the glutaraldehyde molecules and, therefore, building the anchors on the glassy particle surface. Now, such decorated particles are able to fix to the structure the GOx (III in Figure 1B) and the sensor is ready. Glucose oxidase has been used for fabrication of biosensors for quantitative determination of glucose in body fluids, foodstuffs, beverages and fermentation liquor. This immobilized enzyme on a nanomaterial component is fundamental to develop new nanoscale devices for different applications. ${ }^{5,18}$ Therefore, to test the sensibility and the efficiency of our glucose sensor we use different concentrations of glucose and honey aqueous dissolutions. Therefore, pure glucose dissolutions and honey dissolutions $(1 \mathrm{~g} / \mathrm{L})$ with glucose concentration between $0.25 \mathrm{~g} / \mathrm{L}$ to $1.5 \mathrm{~g} / \mathrm{L}$, were employed. Honey dissolution concentration was selected in accordance with Argentine food code $(\mathrm{CAA})^{19}$ that establishes glucose content in honey between $30-35 \%$ $(\mathrm{w} / \mathrm{w})$. Then, a visual inspection was carried out to verify the sensor response using $0.1 \mathrm{~g}$ of MGGS with $5 \mu \mathrm{L}$ of each dissolution (Figure 5 \& Figure 6). No color is observed with a glucose concentration of $0.25 \mathrm{~g} / \mathrm{L}$. However, a slight pink coloration, i.e. a positive reaction indicating the presence of glucose was obtained between 0.4 to $1 \mathrm{~g}$ of glucose per liter. Finally, for concentrations higher than $1.5 \mathrm{~g}$ of glucose / L the color intensity becomes stronger, evidencing that the intensity of color is correlated to the content of glucose. The developed MGGS to detect glucose has two important advantages. One of them is that the color develops in just 60 seconds and the color stabilization takes 120 seconds. On the other hand, the determination is carried out at room temperature unlike the enzymatic method. Therefore, it is a fast, low cost and sensitive proposal for the detection of glucose.

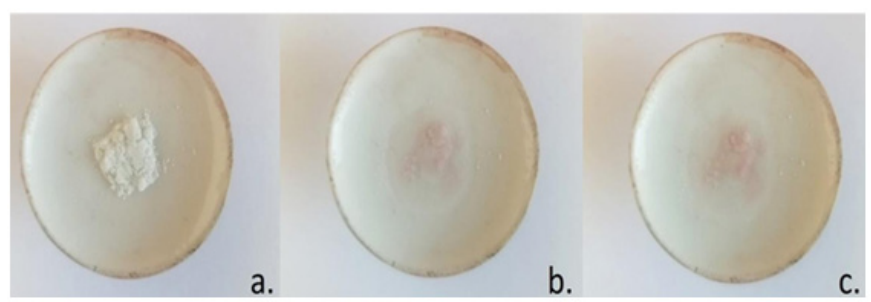

Figure 5 A) Solid sensor; B) Sensor+ Glucose $0.6 \mathrm{~g} / \mathrm{L}$ at $\mathrm{t}=60 \mathrm{sec}$; $\mathrm{C}$. Sensor+ Glucose $0.6 \mathrm{~g} / \mathrm{L}$ at $\mathrm{t}=120 \mathrm{sec}$.

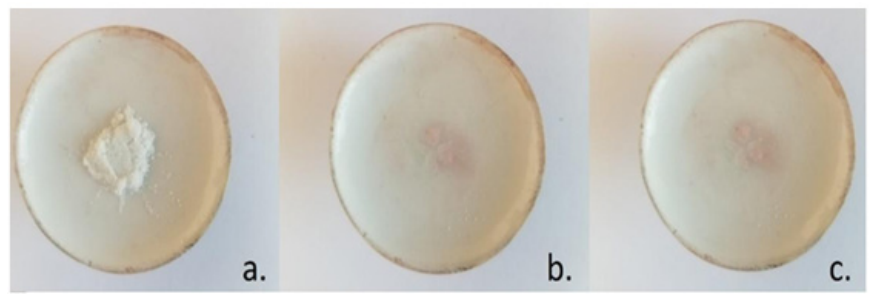

Figure 6 A) Solid sensor; B) Sensor+ Honey I $g / L$ at $t=60$ sec; C. Sensor+ Honey I $\mathrm{g} / \mathrm{L}$ at $\mathrm{t}=120 \mathrm{sec}$.

\section{Conclusion}

In this work a detailed procedure to anchor a glucose biosensor on glassy microparticles is developed using urea medium. This method is fast and effective and avoids the use of expensive chemical substances. Also, the methodology provides useful techniques to guarantee the success of each step. Finally, the screening test on glucose detection in real time was done and also, on a food that may contain glucose, honey. The sensor developed is very simple to use, is portable and only requires a visual inspection to confirm the presence of glucose only through a simple sample aqueous dilution. 


\section{Acknowledgements}

This work has been possible to the financing support by Universidad Nacional del Sur (PGI 24/Q112- Frechero; PGI es 24/ Q097- Centurión) and PUE- INQUISUR CONICET 2017. M.Z. is fellow PUE 2017 CONICET and M.A.F is Researcher Fellow of the CONICET Argentina.

\section{Conflicts of interest}

The author declares that there are no conflicts of interest.

\section{Refrences}

1. Janata J. Principles of Chemical Sensors. New York, London. Plenum Press; 1989.

2. Kisaalita WS. Biosensor standards requirements. Biosensors \& Bioelectronics. 1992;7(9):613-620.

3. Nikhil Bhalla, Pawan Jolly, Nello Formisano, et al. Introduction to biosensors. Essays in Biochemistry. 2016;60:1-8.

4. Wen Z, Ye B, Zhou X. Direct electron transfer reaction of glucose oxidase at bare silver electrodes and its application in analysis. Electroanalysis. 1991;9(8):641-644.

5. Salimi A, Sharifi E, NoorbakhshA, et al. Immobilization of glucose oxidase on electrodeposited nickel oxide nanoparticles: Direct electron transfer and electrocatalytic activity. Biosensors and Bioelectronics. 2007;22:3146-3153.

6. Wang J, Rivas G, Chicharro M. Glucose microsensor based on electrochemical deposition of iridium and glucose oxidase onto carbon fiber electrodes. Electroanal Chem. 1997;439:55-61.

7. Colowick SP, Kaplan N. Immobilized Enzymes, Methods in Enzymology. Immobilized Enzymes. New York. Academic Press; 1976.

8. Gartmann N, Brühwiler D. Functional Group Distributions on Mesoporous Silica. CHIMIA. 2011;65(4):250-252.
9. Limbut W, Thavarungkul P, Kanatharana P, et al. Comparative study of controlled pore glass, silica gel and Poraver ${ }^{\circledR}$ for the immobilization of urease to determine urea in a flow injection conductimetric biosensor system. Biosensors and Bioelectronics. 2004;19:813-821.

10. Gunda NSK, Singh M, Norman L, et al. Optimization and characterization of biomolecule immobilization on silicon substrates using (3-aminopropyl)triethoxysilane (APTES) and glutaraldehyde linker. Applied Surface Science. 2014;305:522-530.

11. Cañas ANA, Macías MPS. Desarrollo de un sistema sensor para la cuantificación de glucosa en jugos de frutas. Rev Soc Quím Méx. 2004;48:106-110.

12. Liu J, Zhang H, Xia X, et al. High sensitivity detection of glucose with negatively charged gold nanoparticles functionalized the gate of AlGaN/ GaN High Electron Mobility Transistor. Sensors and Actuators A: Physical. 2020;312:112128.

13. Yuxuan Gong, Jian Xu, Relva C Buchanan. The aqueous corrosion of nuclear waste glasses revisited: Probing the surface and interfacial phenomena. Corrosion Science. 2018;143:65-75.

14. El-Shamy TM, Pantano CG. Decomposition of silicate glasses in alkaline solutions. Nature. 1977;266:704.

15. Bunker BC. Molecular mechanisms for corrosion of silica and silicate glasses. J Non-Cryst Solids. 1994;179:300.

16. Scholze H. Glass-water interactions. J Non-Cryst Solids. 1988;102:1.

17. Robinet L, Hall C, Eremin K, et al. Alteration of soda silicate glasses by organic pollutants in museums: Mechanisms and kinetics. Journal of Non-Crystalline Solids. 2009;355:1479-1488.

18. Willner I. Biomaterials for Sensors, Fuel Cells, and Circuitry. Science. 2002;98:2407-2408.

19. Código Alimentario Argentino. Alimentos azucarados, Capítulo X. 